\title{
Emergent scenario in the Einstein-Cartan theory
}

\author{
Qihong Huang ${ }^{1}$, Puxun $\mathrm{Wu}^{2,3}$ and Hongwei $\mathrm{Yu}^{1,2, *}$ \\ 1 Institute of Physics and Key Laboratory of Low Dimensional \\ Quantum Structures and Quantum Control of Ministry of Education, \\ Hunan Normal University, Changsha, Hunan 410081, China \\ 2 Center for Nonlinear Science and Department of Physics, \\ Ningbo University, Ningbo, Zhejiang 315211, China \\ ${ }^{3}$ Center for High Energy Physics, Peking University, Beijing 100080, China
}

\begin{abstract}
We study the emergent scenario, which is proposed to avoid the big bang singularity, in the Einstein-Cartan (EC) theory with a positive cosmological constant and a perfect fluid by analyzing the existence and stability of the Einstein static (ES) solutions. We find that there is no stable ES solution for a spatially flat or open universe. However, for a spatially closed universe, the stable ES solution does exist, and in the same existence parameter regions, there also exists an unstable one. With the slow decrease of the equation of state $w$ of the perfect fluid, the stable and unstable critical points move close gradually and coincide once $w$ reaches a critical value, so that the stable critical point becomes an unstable one. As a result, if $w$ approaches a constant at $t \rightarrow-\infty$, the universe can stay at the stable ES state past eternally, and furthermore it can naturally exit from this state and evolve into an inflationary era if $w$ decreases slowly as time goes forward. Therefore, the emergent scenario that avoids the big bang singularity can be successfully implemented in the EC theory of gravity.
\end{abstract}

PACS numbers: $98.80 . \mathrm{Cq}, 04.50 . \mathrm{Kd}$

\footnotetext{
* Corresponding author
} 


\section{INTRODUCTION}

One of the most basic and ancient questions in cosmology is whether the Universe has a beginning or has existed eternally, and this question has been brought into serious discussion by using the knowledge of general relativity and modern cosmology in recent decades. However, the answer is still far from clear. The standard cosmological model implies that the Universe stems from a big bang singularity. To avoid this singularity, Ellis et al.[1, 2] proposed the so-called emergent scenario, in which the Universe stays in an Einstein static (ES) state past eternally and then evolves to a subsequent inflationary era. This scenario suggests that the Universe originates from an ES state rather than a big bang singularity. Unfortunately, the original emergent scenario does not seem to successfully resolve the singularity problem as expected, because there is no stable ES solution in general relativity [3-6] so that the Universe hardly stays at the initial state in a long time as a result of the existence of various perturbations. However, recent studies show that an implementation of the emergent scenario is possible in the modified theories of gravity. In this regard, it has been found that the ES state is stable against homogeneous scalar perturbations in various such theories including $f(R)$ gravity, $f(T)$ gravity, loop quantum gravity, and so on [7-12].

The Einstein-Cartan (EC) theory introduced by Élie Cartan in 1923 [13] is an interesting modified gravity, since it extends, in a natural way, Einstein's general relativity by including the spacetime torsion, instead of assuming it to be zero, and treating it in the same footing as the spacetime curvature. In the EC gravity, in which the metric and the non-symmetric affine connection are two independent quantities, the torsion arises from the matter source of spinor fields, and it is not a dynamical quantity [14-16] since there is a relation between the intrinsic angular momentum (spin) of fermionic matter and the spacetime torsion. Thus, one can incorporate the spinor field into the torsion-free general theory of relativity [17]. As a result, the EC theory is equal to the general relativity with an addition of an effective perfect fluid [18-21] in the energy-momentum tensor, which is the contribution of the spacetime torsion. At macroscopic scales, fermionic particles can be averaged and described as a spin fluid: the Weyssenhoff fluid [18, 19]. The combination of the torsion and the spin fluid in the cosmological context of the EC theory behaves as a stiff matter with a negative energy density [22-24], which leads to gravitational repulsion. This becomes very significant at extremely high densities, and it can help to avoid the curvature singularity by violating the 
energy condition of the singularity theorems [25, 26] and the big bang singularity through a nonsingular big bounce $[27,28]$. The EC theory has also been shown to be able to solve the flatness and horizon problems without an exponential inflation [29-32]. Furthermore, the effects of torsion and the spinning matter in the cosmic inflation, late time acceleration, and so on, have been studied in [24, 26, 33-38].

Recently, the existence and stability of an ES universe in the EC theory with a Weyssenhoff perfect fluid and a normal perfect fluid are studied in [39]. It has been found that there is a stable ES state in a closed spatial geometry and the Universe can stay at this stable state eternally. However, since this state corresponds to a center equilibrium point, our Universe cannot naturally evolve from it into an inflationary era. So it remains unclear whether an emergent scenario that avoids the big bang singularity can be successfully implemented in the EC theory of gravity and this is exactly what we are planing to address in the present paper. We systematically study the existence and stability of the Einstein static state in all possible spatial geometries in the EC theory with a postive cosmological constant rather than only the spatially closed case considered in [39] and in particular we demonstrate that with a positive cosmological constant the emergent scenario can be successfully realized in the EC theory in the spatially closed universe.

The paper is organized as follows. In Section 2, we review the cosmic equations in the framework of the EC theory. In Section 3, we analyze the conditions of the existence of ES solutions, and discuss their stability and the phase transition from the stable ES state to an inflation. Finally, our main conclusion is presented in Section 4. Throughout this paper, unless specified, we adopt the metric signature $(+,-,-,-)$ and set $8 \pi G=\kappa$. Latin indices run from 0 to 3 and the Einstein convention is assumed for repeated indices.

\section{THE FIELD EQUATIONS OF EINSTEIN-CARTAN THEORY}

In the EC theory of gravity, the action takes the form

$$
S=\int \sqrt{-g} d^{4} x\left[-\frac{1}{2 \kappa}(\tilde{R}-2 \Lambda)+\mathcal{L}_{M}\right],
$$

where $\tilde{R}$ is the Ricci scalar constructed by the asymmetric connection $\tilde{\Gamma}_{\alpha \beta}^{\mu}$ rather than the symmetric affine (Levi-Civita) connection $\Gamma_{\alpha \beta}^{\mu}$ used in general relativity, $\Lambda$ is the cosmological constant and $\mathcal{L}_{M}$ is the Lagrangian density of matter. In this theory, the spacetime 
curvature and torsion have the same status with the curvature and torsion tensors being defined respectively by $\tilde{R}_{\nu \lambda \alpha}^{\mu}=\partial_{\lambda} \tilde{\Gamma}_{\nu \alpha}^{\mu}-\partial_{\alpha} \tilde{\Gamma}_{\nu \lambda}^{\mu}+\tilde{\Gamma}_{\beta \lambda}^{\mu} \tilde{\Gamma}_{\nu \alpha}^{\beta}-\tilde{\Gamma}_{\beta \alpha}^{\mu} \tilde{\Gamma}_{\nu \lambda}^{\beta}$ and $T_{\nu \alpha}^{\mu}=\tilde{\Gamma}_{\nu \alpha}^{\mu}-\tilde{\Gamma}_{\alpha \nu}^{\mu}$. And the connection $\tilde{\Gamma}_{\alpha \beta}^{\mu}$ can be decomposed into two parts

$$
\tilde{\Gamma}_{\alpha \beta}^{\mu}=\Gamma_{\alpha \beta}^{\mu}+K_{\alpha \beta}^{\mu}
$$

where $K_{\alpha \beta}^{\mu}$ is the contorsion tensor, which relates to the torsion tensor via

$$
K_{\alpha \beta}^{\mu}=\frac{1}{2}\left(T_{\alpha \beta}^{\mu}-T_{\alpha \beta}^{\mu}-T_{\beta}{ }^{\mu}{ }_{\alpha}\right) .
$$

Varying the action (1) with respect to the metric tensor and the contorsion tensor, respectively, gives the field equations of the EC theory [14-16]

$$
\begin{aligned}
G^{\mu \nu}-\Lambda g^{\mu \nu}-\left(2 T_{\alpha \beta}^{\beta}+\tilde{\nabla}_{\alpha}\right)\left(Q^{\mu \nu \alpha}-Q^{\nu \alpha \mu}-Q^{\alpha \mu \nu}\right) & =\kappa T^{\mu \nu}, \\
Q^{\mu \nu \alpha} & =\kappa \tau^{\mu \nu \alpha},
\end{aligned}
$$

where $G^{\mu \nu}$ is the Einstein tensor, $Q_{\mu \nu}{ }^{\alpha}=T_{\mu \nu}{ }^{\alpha}+\delta_{\mu}^{\alpha} T_{\nu \beta}{ }^{\beta}-\delta_{\nu}^{\alpha} T_{\mu \beta}{ }^{\beta}, T^{\mu \nu}=\frac{2}{\sqrt{-g}} \frac{\delta \mathcal{L}_{m}}{\delta g_{\mu \nu}}$ is the energy-momentum tensor, and $\tau^{\mu \nu \alpha}=\frac{1}{\sqrt{-g}} \frac{\delta \mathcal{L}_{m}}{\delta K_{\mu \nu \alpha}}$ is the spin density tensor. Eq. (5) shows that the torsion is proportional to the spin density, which indicates that the latter is the source of the former.

Combining Eqs. (4) and (5), one can show that the field equations can be expressed in the standard form of general relativity with a modification in the energy-momentum tensor [1921] as

$$
G^{\mu \nu}-\Lambda g^{\mu \nu}=\kappa\left(T^{\mu \nu}+\theta^{\mu \nu}\right)
$$

where

$$
\theta^{\mu \nu}=-4 \tau_{[\beta}^{\mu \alpha} \tau_{\alpha]}^{\nu \beta}-2 \tau^{\mu \alpha \beta} \tau_{\alpha \beta}^{\nu}+\tau^{\alpha \beta \mu} \tau_{\alpha \beta}{ }^{\nu}+\frac{1}{2} g^{\mu \nu}\left(4 \tau_{\lambda}{ }_{[\beta}{ }^{\lambda \beta}{ }_{\alpha]}{ }^{\lambda \beta}+\tau^{\alpha \beta \lambda} \tau_{\alpha \beta \lambda}\right)
$$

is the correction to the energy-momentum tensor generated by the torsion.

In the EC theory, $T^{\mu \nu}$ can be separated as follows

$$
T^{\mu \nu}=T_{F}^{\mu \nu}+T_{S}^{\mu \nu}
$$

Here, $T_{F}^{\mu \nu}=(\rho+p) u^{\mu} u^{\nu}-p g^{\mu \nu}$ represents the usual perfect fluid with $u_{\mu}$ being the four velocity, and $\rho$ and $p$ being the energy density and pressure, respectively. Assuming its 
equation of state is a constant, one has $p=w \rho . T_{S}^{\mu \nu}$ is the energy-momentum tensor of an intrinsic-spin fluid, which reads

$$
\begin{aligned}
T_{S}^{\mu \nu}= & u^{(\mu} S^{\nu) \alpha} u^{\beta} u_{\alpha ; \beta}+\left(u^{(\mu} S^{\nu) \alpha}\right)_{; \alpha}+T_{\alpha \beta}{ }^{(\mu} u^{\nu)} S^{\beta \alpha} \\
& -u^{\beta} S^{\alpha(\nu} T_{\alpha \beta}^{\mu)}-\omega^{\alpha(\mu} S_{\alpha}^{\nu)}+u^{(\mu} S^{\nu) \alpha} \omega_{\alpha \beta} u^{\beta},
\end{aligned}
$$

where $\omega$ is the angular velocity associated with the intrinsic spin and a semicolon represents the covariant derivative with respect to the Levi-Civita connection. This spin fluid can be described by the so-called Weyssenhoff fluid, which is a continuous macroscopic medium characterized in microscopic scales by the spin of matter fields. The spin density of the Weyssenhoff fluid is described by an antisymmetric tensor $S_{\mu \nu}=-S_{\nu \mu}$ and has the form [18]

$$
\tau_{\nu \alpha}^{\mu}=u^{\mu} S_{\nu \alpha}
$$

Substituting this expression into Eqs. $(7,9)$, one can obtain that [19-21]

$$
\begin{array}{r}
T_{S}^{\mu \nu}=-\kappa \sigma^{2} u^{\mu} u^{\nu} \\
\theta^{\mu \nu}=\frac{1}{2} \kappa \sigma^{2} u^{\mu} u^{\nu}+\frac{1}{4} \kappa \sigma^{2} g^{\mu \nu}
\end{array}
$$

where

$$
\sigma^{2}=\frac{1}{2} S_{\mu \nu} S^{\mu \nu}
$$

is the spin density scalar.

Thus, rewriting the field equations of the EC theory as

$$
G^{\mu \nu}=\kappa \tilde{T}^{\mu \nu}
$$

we have

$$
\tilde{T}^{\mu \nu}=T^{\mu \nu}+\theta^{\mu \nu}=\left(\rho+p-\rho_{s}-p_{s}\right) u^{\mu} u^{\nu}-\left(p-p_{s}-\kappa^{-1} \Lambda\right) g^{\mu \nu}
$$

where $\rho_{s}=p_{s}=\frac{1}{4} \kappa \sigma^{2}$. Since $p_{s} / \rho_{s}=1$, the effect of torsion and the spin matter can be considered as a stiff matter with negative energy density and pressure, which leads to gravitational repulsion. Here we can see that the correction to the energy-momentum tensor due to the spin-spin interaction is of the second order in the gravitational coupling constant $(\kappa)$. Thus, it is extremely weak, but it can become very significant at extremely high energy densities in the very early universe. 
To study the ES solution in the EC theory, we consider a homogeneous and isotropic universe described by the Friedman-Lemaitre-Robertson-Walker metric:

$$
d s^{2}=d t^{2}-a^{2}(t)\left[\frac{d r^{2}}{1-k r^{2}}+r^{2}\left(d \theta^{2}+\sin ^{2} \theta d \phi^{2}\right)\right],
$$

where $t$ is the cosmic time and $a(t)$ is the cosmic scale factor. $k=0,1$ or -1 correspond to a spatially flat, closed or open universe, respectively. Substituting this metric into the field equations (Eq. (14)), we find that the $0-0$ component gives the Friedmann equation

$$
\dot{a}^{2}+k=\frac{\kappa}{3}\left(\rho-\rho_{s}\right) a^{2}+\frac{\Lambda}{3} a^{2},
$$

where a dot denotes the derivative with respect to the cosmic time $t$. Since the perfect fluid satisfies the continuity equation $\dot{\rho}+3 \frac{\dot{a}}{a}(1+w) \rho=0$ and $\tilde{T}_{; \mu}^{\mu \nu}=0$, one can obtain that $[23,39]$

$$
\rho=\rho_{0} a^{-3(1+w)}, \quad \rho_{s}=\tilde{D} a^{-6} .
$$

Here $\rho_{0}$ and $\tilde{D}$ are two integral constants, which are assumed to be positive. By differentiating the Friedmann equation and using Eq. (18), we get the Raychadhuri equation

$$
2 \ddot{a}=-(1+3 w) \frac{\dot{a}^{2}+k}{a}+D(1-w) \frac{1}{a^{5}}+\Lambda(1+w) a,
$$

where $D \equiv \kappa \tilde{D}$.

\section{THE EINSTEIN STATIC SOLUTIONS AND THEIR STABILITY}

The ES solution is given by the condition $\ddot{a}=\dot{a}=0$, which implies $a=a_{E s}$ and $H\left(a_{E s}\right)=$ 0 . Thus, the Raychadhuri equation dictates that the critical points are determined by the following cubic equation of $\frac{1}{a_{E s}^{2}}$

$$
(1-w) D \frac{1}{a_{E s}^{6}}-(1+3 w) \frac{k}{a_{E s}^{2}}+\Lambda(1+w)=0 .
$$

And the corresponding energy density $\rho$ at the critical point satisfies

$$
\kappa \rho\left(a_{E s}\right)=\frac{3 k}{a_{E s}^{2}}+\frac{D}{a_{E s}^{6}}-\Lambda,
$$

which is obtained from the Friedmann equation. Since $a_{E s}$ is positive and $\rho\left(a_{E s}\right)$ should take a nonnegative value, the existence conditions of the critical point are $\rho\left(a_{E s}\right) \geq 0$ and $a_{E s}^{2}>0$. 
In order to simplify our discussion, we rewrite Eqs. $(20,21)$ as follows:

$$
\begin{gathered}
(1-w) D_{0} \frac{1}{\left(\Lambda a_{E s}^{2}\right)^{3}}-(1+3 w) \frac{k}{\Lambda a_{E s}^{2}}+(1+w)=0 . \\
\kappa \rho\left(a_{E s}\right)=\left[\frac{3 k}{\Lambda a_{E s}^{2}}+\frac{D D_{0}}{\left(\Lambda a_{E s}^{2}\right)^{3}}-1\right] \Lambda,
\end{gathered}
$$

where $D_{0}=D \Lambda^{2}>0$. For a cubic equation given in (22), the solutions are determined by the following expression

$$
\Delta=B^{2}-4 A C
$$

where $A=-3 \lambda \alpha, B=-9 \lambda \beta$, and $C=\alpha^{2}$ with $\lambda=(1-w) D_{0}, \alpha=-(1+3 w) k$ and $\beta=(1+w)$. Respectively, $\Delta>0,=0$ and $<0$ correspond to one, two, and three real solution(s).

To study the stability of critical points, we introduce two variables $x_{1}=a$ and $x_{2}=\dot{a}$, and as a result, we have

$$
\begin{gathered}
\dot{x}_{1}=x_{2}, \\
2 \dot{x}_{2}=-(1+3 w) \frac{x_{2}^{2}+k}{x_{1}}+(1-w) D \frac{1}{x_{1}^{5}}+\Lambda(1+w) x_{1} .
\end{gathered}
$$

The stability of these critical points are determined by the eigenvalues of the coefficient matrix, which are obtained from linearizing the system described by the above equations near these critical points. The eigenvalue $\mu^{2}$ can be expressed as:

$$
\mu^{2}=\left[\frac{1+3 w}{2} \frac{k}{\Lambda a_{E s}^{2}}-\frac{5}{2}(1-w) D_{0} \frac{1}{\left(\Lambda a_{E s}^{2}\right)^{3}}+\frac{1}{2}(1+w)\right] \Lambda .
$$

If $\mu^{2}<0$, a small perturbation from the fixed point will result in an oscillation about this point rather than a exponential deviation. Thus, the corresponding ES solution is a center equilibrium point. Otherwise, it is an unstable point.

For the case $\Lambda=0$, from Eqs. (20, 21, 27), we find that

$$
a_{E s}^{4}=\frac{(1-w) D}{(1+3 w) k}, \quad \kappa \rho\left(a_{E s}\right)=\frac{1}{a_{E s}^{2}} \frac{4 k}{1-w}, \quad \mu^{2}=\frac{2 D}{a_{E s}^{6}}(w-1) .
$$

So there exists a critical point which is stable under the condition $D>0$ and $-\frac{1}{3}<w<1$ for $k=1$, as has been studied in [39]. Apparently, the universe can stay at this ES state eternally, but cannot exit from this state and naturally evolve into an inflationary phase. The critical point is unstable for $k=-1$, although it exists under the condition $w>1$ and $D>0$. 
Now, we discuss the case with a positive cosmological constant. We consider all possible spatial geometries, i.e., the spatial flat, closed and open universes rather than only the closed one with $k=1$.

A. $k=0$

For a spatially flat universe $(k=0)$, Eqs. (22) and (23) reduce to

$$
\frac{D_{0}}{\left(\Lambda a_{E s}^{2}\right)^{3}}=\frac{1+w}{-1+w}, \quad \kappa \rho\left(a_{E s}\right)=\frac{2}{-1+w} \Lambda,
$$

which show that the existence condition for the critical point is $w>1$. From Eq. (27), one can see that the eigenvalue $\mu^{2}=3(1+w) \Lambda$ is positive in the existence region, and this means that there is no stable ES solution in this case.

B. $k=1$

For a spatially closed universe $(k=1)$, we have $\lambda=(1-w) D_{0}, \alpha=-(1+3 w)$ and $\beta=(1+w)$. To analyze the solutions of Eq. (22), we need to consider the value of $\Delta$ in three different cases.

(i) $\Delta>0$ : This requires $w>1$, or $w<-\frac{1}{3}$, or $-\frac{1}{3}<w<1$ with $D_{0}>-\frac{4(1+3 w)^{3}}{27(-1+w)(1+w)^{2}}>$ 0 . The only one real solution of Eq. (22) in this case, which we label as Point E, can be expressed as

$$
\text { Point } E: \frac{1}{\Lambda a_{E s}^{2}}=-\frac{1}{3 \lambda}\left[Y_{+}^{\frac{1}{3}}+Y_{-}^{\frac{1}{3}}\right],
$$

where $Y_{ \pm}=\frac{3 \lambda}{2}(-B \pm \sqrt{\Delta})$. Apparently, $\Lambda a_{E s}^{2}>0$ requires that $\lambda<0$ and both $Y_{+}$and $Y_{-}$ are positive. For the case of $\lambda<0, w>1$ is needed, and this leads to $B>0$ and $A<0$. One can see that $Y_{+}=\frac{3 \lambda}{2}(-B+\sqrt{\Delta})<0$ since $\Delta>B^{2}$. This conflicts with the requirement of $Y_{+}>0$ from $\Lambda a_{E s}^{2}>0$. Thus, the critical point $E$ is physically meaningless and should be discarded.

(ii) $\Delta<0$ : In this case

$$
0<D_{0}<-\frac{4(1+3 w)^{3}}{27(-1+w)(1+w)^{2}}, \quad-\frac{1}{3}<w<1
$$

are required, and these yield $\lambda>0, B<0$ and $A>0$. Now Eq. (22) has three different real 
solutions and thus there exist three different critical points:

$$
\begin{gathered}
\text { Point } F: \quad \frac{1}{\Lambda a_{E s}^{2}}=-\frac{2}{3 \lambda} \sqrt{A} \cos \left(\frac{\theta}{3}\right), \\
\text { Point } G: \frac{1}{\Lambda a_{E s}^{2}}=\frac{\sqrt{A}}{3 \lambda}\left[\cos \left(\frac{\theta}{3}\right)+\sqrt{3} \sin \left(\frac{\theta}{3}\right)\right], \\
\text { Point } \quad H: \quad \frac{1}{\Lambda a_{E s}^{2}}=\frac{\sqrt{A}}{3 \lambda}\left[\cos \left(\frac{\theta}{3}\right)-\sqrt{3} \sin \left(\frac{\theta}{3}\right)\right],
\end{gathered}
$$

where $\theta=\arccos (T)$ with $T=-\frac{3}{2} \lambda B A^{-\frac{3}{2}}$. It is easy to see that $0<T<1$, and as a result, $0<\theta<\frac{\pi}{2}$. Since $\lambda>0, \Lambda a_{E s}^{2}<0$ for point $F$. Thus, this point is not physically meaningful.

In the region $0<\theta<\frac{\pi}{2}, \cos \left(\frac{\theta}{3}\right)>\sqrt{3} \sin \left(\frac{\theta}{3}\right)>0$, and consequently $\Lambda a_{E s}^{2}$ for critical points $G$ and $H$ are positive. In addition, we find that if conditions given in (31) are obeyed, $\rho\left(a_{E s}\right)$ is also positive for these two critical points. Thus, both points $G$ and $H$ are physical under the conditions given in (31).

(iii) $\Delta=0$ : This implies that $D_{0}=-\frac{4(1+3 w)^{3}}{27(-1+w)(1+w)^{2}}$ with $-\frac{1}{3}<w<1$. In this case, there are two different real solutions. We find that critical points $G$ and $H$ coincide because of $\theta=0$. Thus, two different critical points are point $F$ with $\theta=0$ and point $G$ or $H$ with $\theta=0$. As in the case of $\Delta<0$, point $F$ is still physically meaningless since it implies $\Lambda a_{E s}^{2}<0$, and as a result, only point $G$ with $\theta=0$ needs to be considered.

Now, we discuss the stability of critical points $G$ and $H$ by analyzing their eigenvalues shown in Eq. (27). We find by numerical calculations that only point $G$ is always stable as long as it exists, and the regions of stability for Point $G$ in the $\left(w, D_{0}\right)$ parameter space are shown in Fig. (1). Point $H$ is always unstable. A summary of the existence and stability of all critical points is given in Tab. (I).

Thus, if our Universe stays at point $G$ initially, it can stay at this state past eternally since it is a stable center point. Now we want to make an estimate about the size of the Universe at this initial stable state. For this purpose, let us note that, with Eq. (33), the scale factor in this stable state can be written as

$$
a_{E s}=\left(D \frac{3(1-w)}{3 w+1}\right)^{1 / 4}\left[\cos \left(\frac{\theta}{3}\right)+\sqrt{3} \sin \left(\frac{\theta}{3}\right)\right]^{-1 / 2} \text {. }
$$

To estimate the value of $a_{E s}$, we take $D_{0}=1$ and $w=0.6$, which are in stable region shown in Fig. (1), and then we get

$$
a_{E s} \simeq \frac{1}{\sqrt{\Lambda}}
$$




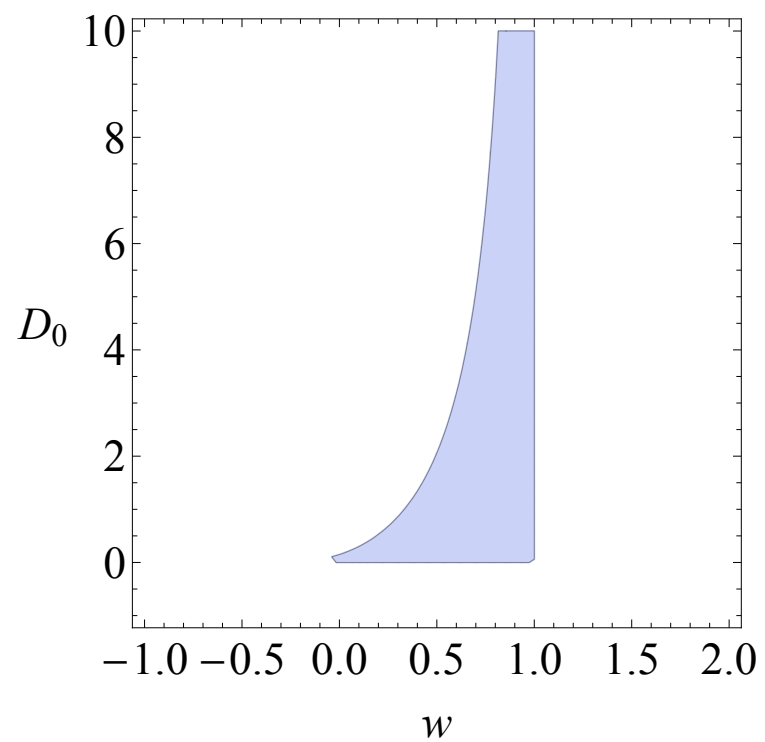

FIG. 1: Regions of stability for point $G$ in the $\left(w, D_{0}\right)$ parameter space.

TABLE I: Summary of the existence and stability of critical points for $k=1$.

\begin{tabular}{|r|r|r|r|}
\hline \hline & Critical point & Existence & Stability \\
\hline$\Delta>0$ & Point $E$ & no & - \\
\hline$\Delta<0$ & Point $F$ & no & - \\
& Point $G$ & yes & stable if it exists \\
& Point $H$ & yes & unstable \\
\hline$\Delta=0$ & Point $F$ with $\theta=0$ & no & - \\
& Point $G$ or $H$ with $\theta=0$ & yes & unstable \\
\hline \hline
\end{tabular}

Assuming $\rho_{\Lambda}=\kappa^{-1} \Lambda$ to be the inflation energy scale $\left(\sim 10^{90} \mathrm{~g} / \mathrm{cm}^{3}\right)$, one has $a_{E s} \sim 10^{-31} \mathrm{~cm}$, which is larger than the Planck length by about two orders of magnitude. If the initial value of the cosmic scale factor deviates slightly from the value given by Eq. (35), the Universe will undergo an infinite oscillation around the center equilibrium point, as shown in Fig. (2). However, a successful emergent scenario that avoids the big bang singularity demands not only a stable ES state that the Universe can stay past eternally but also a natural exit from it into an inflationary era. Next we show that this is possible. For this purpose, we assume that the equation of state of the perfect fluid approaches a constant at $t \rightarrow-\infty$ 


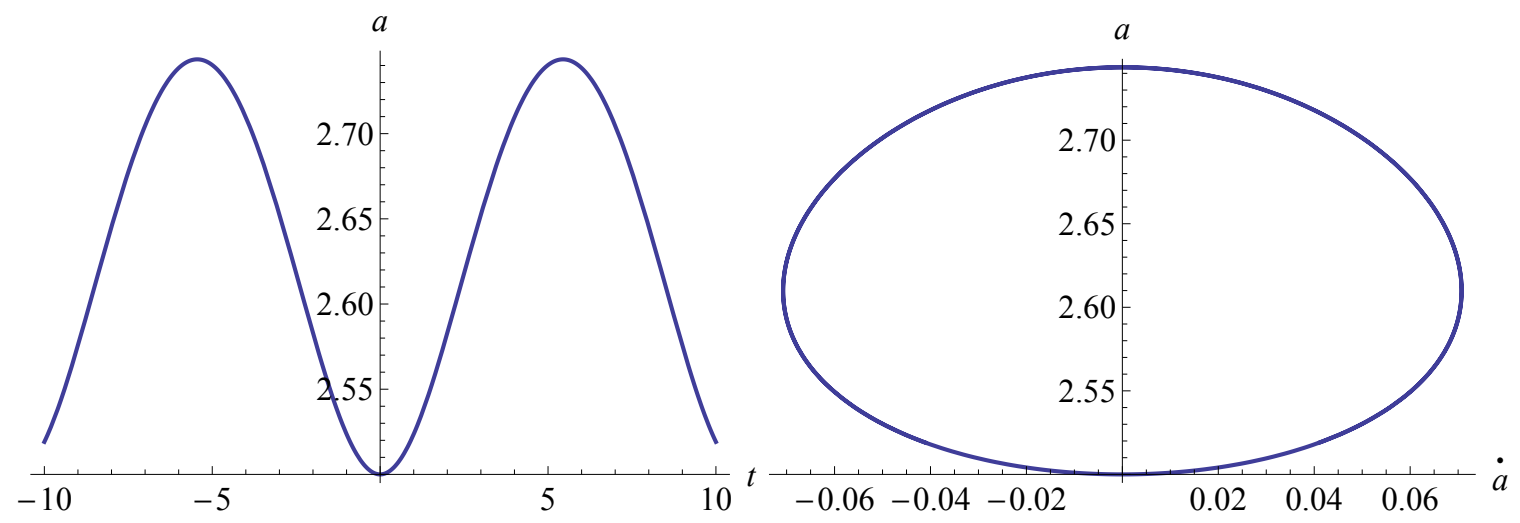

FIG. 2: Evolutionary curve of the scale factor with time and the corresponding phase diagram in space $(a, \dot{a})$ with $w=0.6, \Lambda=0.1$ and $D_{0}=2$.

and decreases very slowly with time going forward. With the decrease of $w$, the stable critical point $G$ and unstable point $H$ will move close gradually and coincide once $w$ reaches a critical value in which $\Delta=0$. So, the stable point $G$ becomes an unstable one. In other words, if the cosmic scale factor satisfies the initial condition which is given in Eq. (35), our Universe can evolve from a stable state to an unstable one with the decrease of $w$, as shown in the left panel of Fig. (3). As a result, the Universe can naturally exit from the stable state and enter an inflationary phase. In the right panel of Fig. (3) we show this phase transition. Finally, let us note that our of analysis of the emergent scenario does not take the quantum effects of gravity into consideration which presumably come into play at very high energies in the very early universe. Fortunately, in the emergent scenario we just described here, the Universe starts from an initial stable state which is about two orders of magnitude larger than the Planck length, so the effects from the quantization of the gravitational field may not be significant and can be safely neglected.

C. $k=-1$

In this case, one has $\lambda=(1-w) D_{0}, \alpha=(1+3 w)$ and $\beta=(1+w)$. 

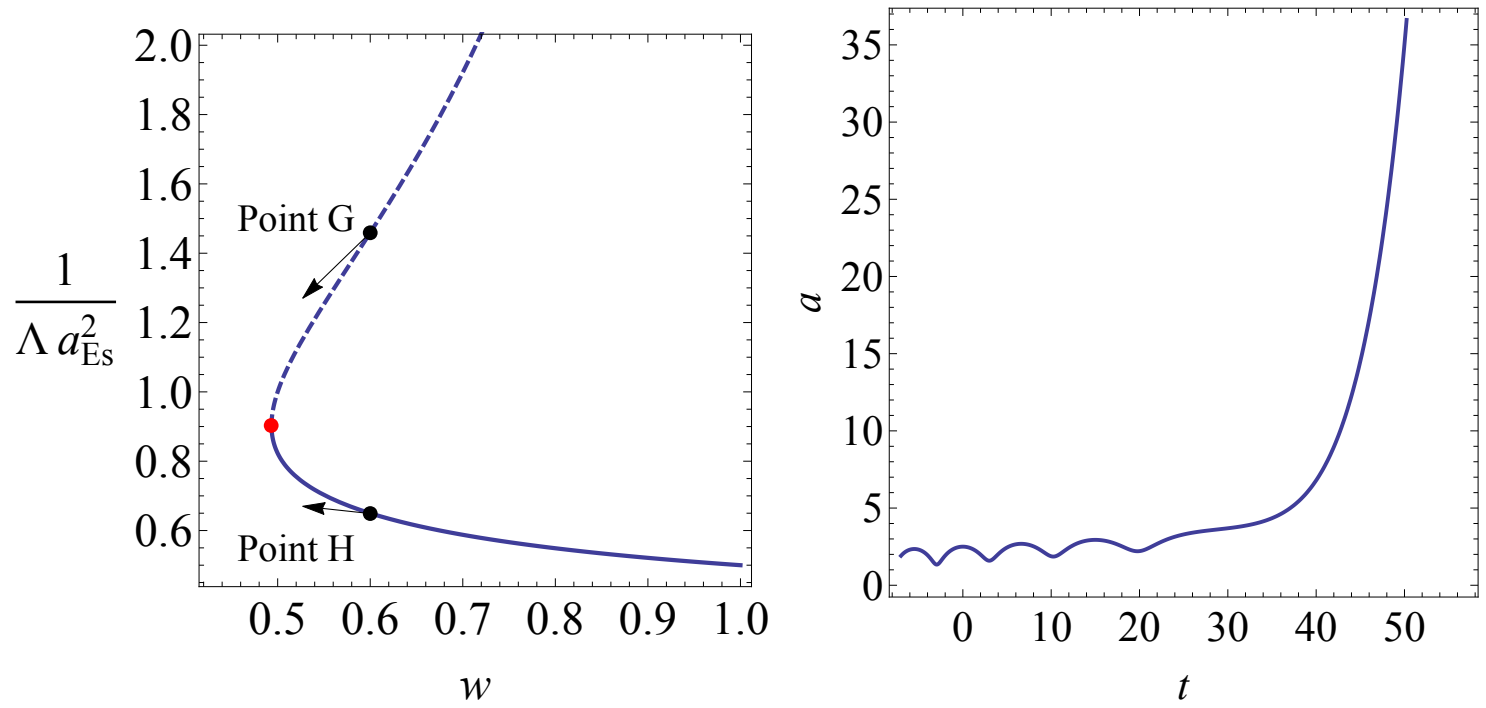

FIG. 3: The evolutions of the stable point $G$ and unstable point $H$ with $D_{0}=2$ and the decrease of $w$ are shown in the left panel, while the right panel shows the phase transition from a stable state to an inflationary one with $D_{0}=2, \Lambda=0.1$ and a slowly decreasing $w$.

(i) $\Delta>0$ : This leads to

$$
\begin{aligned}
& D_{0}>\frac{4(1+3 w)^{3}}{27(-1+w)(1+w)^{2}}>0, \quad|w|>1, \\
& D_{0}>\frac{4(1+3 w)^{3}}{27(-1+w)(1+w)^{2}}>0, \quad-1<w<-\frac{1}{3} \text {, } \\
& \text { or } \quad-\frac{1}{3}<w<1 .
\end{aligned}
$$

There is one real solution, labeled as Point $I$, which has the same form as Point $E$. As have been discussed in the case of $k=1, \Lambda a_{E s}^{2}>0$ requires that $\lambda<0$ and both $Y_{+}$and $Y_{-}$are positive. Since $\lambda<0$ means $w>1$, which gives $B>0$ and $A>0$, both $Y_{+}$and $Y_{-}$are positive. We find that if the conditions

$$
w>1, \quad D_{0}>\frac{4(1+3 w)^{3}}{27(-1+w)(1+w)^{2}}>0
$$

are satisfied, $\rho\left(a_{E s}\right)$ is also positive. Thus, Eq. (40) gives the existence condition for Point $I$.

(ii) $\Delta<0$ : This inequality leads to $0<D_{0}<\frac{4(1+3 w)^{3}}{27(-1+w)(1+w)^{2}}$ with $|w|>1$ or $-1<w<-\frac{1}{3}$. Eq. (22) has three real solutions in this case. We name them as Points $J, K$ and $L$, which have the same forms as Points $F, G$ and $H$. One can obtain that $A>0$ and $-1<T<1$, which imply $0<\theta<\pi, \cos \left(\frac{\theta}{3}\right)>0$ and $\sin \left(\frac{\theta}{3}\right)>0$. 
For Point $J, \Lambda a_{E s}^{2}>0$ requires $\lambda<0$. If $0<D_{0}<\frac{4(1+3 w)^{3}}{27(-1+w)(1+w)^{2}}$ and $w>1$ are satisfied, both $\Lambda a_{E s}^{2}$ and $\rho\left(a_{E s}\right)$ are positive. Thus, different from Point $F$, this critical point is physically meaningful.

For Point $K$, the $\Lambda a_{E s}^{2}>0$ condition requires $0<D_{0}<\frac{4(1+3 w)^{3}}{27(-1+w)(1+w)^{2}}$ with $-1<w<-\frac{1}{3}$

or $w<-1$, while the one for Point $L$ demands $0<D_{0}<\frac{4(1+3 w)^{3}}{27(-1+w)(1+w)^{2}}$ with $-1<w<-\frac{1}{3}$. $\rho\left(a_{E s}\right)$ for Points $K$ and $L$ are negative when $\Lambda a_{E s}^{2}>0$. Thus, these two critical points are not physical.

(iii) $\Delta=0$ : This means that $D_{0}=\frac{4(1+3 w)^{3}}{27(-1+w)(1+w)^{2}}$ with $-1<w<-\frac{1}{3}$ or $|w|>1$. There are two different real solutions which are Point $J$ with $\theta=0$ and Point $K$ or $L$ with $\theta=0$. As in the case of $\Delta<0$, Point $J$ is physical under the condition $w>1$ because of $\Lambda a_{E s}^{2}=-\frac{2 \sqrt{A}}{3 \lambda}>0$ and $\kappa \rho\left(a_{E s}\right)=\frac{2(7+9 w) \Lambda}{(-1+w)(1+3 w)}>0$. For Point $K$ or $L$ with $\theta=0$, the requirements from $\Lambda a_{E s}^{2}>0$ and $\rho\left(a_{E s}\right)>0$ conflict with each other and so this critical point is not physical.

In summary, in the case of $k=-1$, Point $I$, Point $J$ and Point $J$ with $\theta=0$ are all physical, but none of them is stable. Thus, there is no stable ES solution in this case.

\section{CONCLUSION}

The EC theory is a modification of Einstein's general relativistic theory of gravitation by introducing spacetime torsion. However, the spacetime torsion is not a dynamical quantity and it can be expressed completely in terms of the spin sources. The EC theory of gravity can be expressed as the general relativity theory with an exotic stiff perfect fluid. Recently, the existence and stability of ES universe in the EC theory with a positive spatial curvature have been discussed in [39], and it has been found that a stable ES solution exists so that the universe can stay at the stable state eternally. This suggests that the EC theory supports a non-singular cosmology. However, this cosmology is not realistic though non-singular, since the universe can not exit from the stable state and naturally evolve into an inflationary era.

In this paper, we reanalyze the existence and stability of ES solutions in the EC theory by adding a positive cosmological constant. In addition to the case of a positive spatial curvature, we also study the cases of $k=0$ and -1 . We find that in spatially flat and open universes there is no stable ES solution. While, for the spatially closed universe $(k=1)$, the 
stable ES solution exists, and in the same parameter regions there also exists an unstable one. With the slow decrease of the equation of state $w$ of the perfect fluid, the stable and unstable critical points move close gradually. Once $w$ reaches a critical value, as shown in the left panel of Fig. (3), the stable critical point coincides with the unstable one, and becomes an unstable point. Thus, if $w$ is a constant at $t \rightarrow-\infty$, the Universe can stay at the stable ES state past eternally. Then, it can naturally evolve into an inflationary era with a slowly decreasing $w$. Therefore, in the EC theory, a successful emergent scenario to avoid the big bang singularity can be successfully implemented.

\section{Acknowledgments}

This work was supported by the National Natural Science Foundation of China under Grants No. 11175093, No. 11222545, No. 11435006, and No. 11375092; the Specialized Research Fund for the Doctoral Program of Higher Education under Grant No. 20124306110001.

[1] G. F. R. Ellis and R. Maartens, Class. Quant. Grav. 21, 223 (2004).

[2] G. F. R. Ellis, J. Murugan, and C. G. Tsagas, Class. Quant. Grav. 21, 233 (2004).

[3] A. S. Eddington, Mon. Not. Roy. Astron. Soc. 90, 668 (1930).

[4] G. W. Gibbons, Nucl. Phys. B 292, 784 (1987).

[5] G. W. Gibbons, Nucl. Phys. B 310, 636 (1988).

[6] J. D. Barrow, G. F. R. Ellis, R. Maartens and C. G. Tsagas, Class. Quant. Grav. 20, L155 (2003).

[7] D. J. Mulryne, R. Tavakol, J. E. Lidsey and G. F. R. Ellis, Phys. Rev. D 71, 123512 (2005); J. E. Lidsey, D. J. Mulryne, N. J. Nunes and R. Tavakol, Phys. Rev. D 70, 063521 (2004); L. Parisi, M. Bruni, R. Maartens and K. Vandersloot, Class. Quant. Grav. 24, 6243 (2007); P. Wu, S. Zhang and H. Yu, JCAP 05, 007 (2009); S. Bag, V. Sahni, Y. Shtanov and S. Unnikrishnan, JCAP 07, 034 (2014).

[8] K. Zhang, P. Wu and H. Yu, Phys. Lett. B 690, 229 (2010); Phys. Rev. D 85, 043521 (2012); J. E. Lidsey and D. J. Mulryne, Phys. Rev. D 73, 083508 (2006); A. Gruppuso, E. Roessl and 
M. Shaposhnikov, JHEP 08, 011 (2004); L.A. Gergely and R. Maartens, Class. Quant. Grav. 19, 213 (2002); K. Atazadeh, Y. Heydarzade, and F. Darabi, Phys. Lett. B 732, 223 (2014); K. Zhang, P. Wu and H. Yu, JCAP 01, 048 (2014); P. Wu and H. Yu, Phys. Lett. B 703, 223 (2011); J. Li, C. Lee and C. Geng, Eur. Phys. J. C 73, 2315 (2013).

[9] P. Wu and H. Yu, Phys. Rev. D 81, 103522 (2010); C. G. Böhmer and F. S. N. Lobo, Eur. Phys. J. C 70, 1111 (2010); K. l. Maeda, Y. Misonoh and T. Kobayashi, Phys. Rev. D 82, 064024 (2010); M. Park, JHEP 09, 123 (2009); R. Canonico and L. Parisi, Phys. Rev. D 82, 064005 (2010); L. Parisi, N. Radicella and G. Vilasi, Phys. Rev. D 86, 024035 (2012); K. Zhang, P. Wu and H. Yu, Phys. Rev. D 87, 063513 (2013); C. G. Böhmer and F. S. N. Lobo, Phys. Rev. D 79, 067504 (2009); C. G. Böhmer, L. Hollenstein, F. S. N. Lobo and S. S. Seahra, arXiv:1001.1266.

[10] S. Carneiro and R. Tavakol, Phys. Rev. D 80, 043528 (2009); A. Odrzywolek, Phys. Rev. D 80, 103515 (2009); C. G. Böhmer, Class. Quant. Grav. 21, 1119 (2004); R. Goswami, N. Goheer and P. K. S. Dunsby, Phys. Rev. D 78, 044011 (2008); C. G. Böhmer, F. S. N. Lobo and N. Tamanini, Phys. Rev. D 88, 104019 (2013); T. Clifton and J. D. Barrow, Phys. Rev. D 72, 123003 (2005); J. D. Barrow and C. G. Tsagas, Class. Quant. Grav. 26, 195003 (2009); A. Vilenkin, Phys. Rev. D 88, 043516 (2013); A. Aguirre and J. Kehayias, Phys. Rev. D 88, 103504 (2013); A. T. Mithani and A. Vilenkin, JCAP 01, 028 (2012); A. T. Mithani and A. Vilenkin, arXiv:1204.4658.

[11] Y. Cai, Y. Wan, and X. Zhang, Phys. Lett. B 731, 217 (2014); Y. Cai, M. Li and X. Zhang, Phys. Lett. B 718, 248 (2012); Z. Liu and Y. Piao, Phys. Lett. B 718, 734 (2013); S. Campo, R. Herrera, and P. Labraña, JCAP 11, 030 (2007); S. Campo, R. Herrera, and P. Labraña, JCAP 07, 006 (2009); H. Huang, P. Wu, and H. Yu, Phys. Rev. D 89, 103521 (2014); Phys. Rev. D 91, 023507 (2015); C. G. Böhmer, F. S. N. Lobo and N. Tamanini, Phys. Rev. D 88, 104019 (2013).

[12] C. G. Böhmer, L. Hollenstein, and F. S. N. Lobo, Phys. Rev. D 76, 084005 (2007); N. Goheer, R. Goswami and P. K. S. Dunsby, Class. Quant. Grav. 26, 105003 (2009); S. S. Seahra and C. G. Böhmer, Phys. Rev. D 79, 064009 (2009); Y. Heydarzade and F. Darabi, arXiv:1501.02624.

[13] E. Cartan, Ann. Ec. Norm. Sup. 40, 325 (1923).

[14] F. W. Hehl, P. von der Heyde, G. D. Kerlick and J. M. Nester, Rev. Mod. Phys. 48, 393 (1976). 
[15] F. W. Hehl, Found. Phys. 15, 451 (1985).

[16] F. W. Hehl, J. D. McCrea, E. W. Mielke and Y. Ne'eman, Phys. Rep. 258, 1 (1995).

[17] T. Watanabe and M. J. Hayashi, General relativity with torsion, gr-qc/0409029; M. A. Lledo and L. Sommovigo, Class. Quant. Grav. 27, 065014 (2010).

[18] Y. N. Obukhov and V. A. Korotky, Class Quantum Gravit. 4, 1633 (1987).

[19] J. Weyssenhoff and A. Raabe, Acta Phys. Pol. 9, 7 (1947).

[20] G. de Berredo-Peixoto and E. A. De Freitas, Int. J. Mod. Phys. A 24, 1652 (2009).

[21] S. D. Brechet, M. P. Hobson and A. N. Lasenby, Class. Quant. Grav. 24, 6329 (2007).

[22] F. W. Hehl, P. von der Heyde and G. D. Kerlick, Phys. Rev. D 10, 1066 (1974).

[23] I. S. Nurgaliev and W. N. Ponomariev, Phys. Lett. 130B, 378 (1983).

[24] M. Gasperini, Phys. Rev. Lett. 56, 2873 (1986).

[25] A. Trautman, Nature 242,7 (1973); J. M. Stewart and P. Hajicek, Nature 244, 96 (1973).

[26] B. Kuchowicz, Gen. Relativ. Gravit. 9, 511 (1978).

[27] N. J. Poplawski, Gen. Relativ. Gravit. 44, 1007 (2012).

[28] A. H. Ziaie, P. V. Moniz, A. Ranjbar and H. R. Sepangi, Eur. Phys. J. C 74, 3154 (2014).

[29] N. J. Poplawski, Phys. Lett. B 694, 181 (2010).

[30] N. J. Poplawski, Phys. Rev. D 85, 107502 (2012).

[31] N. J. Poplawski, Annalen Phys. 523, 291 (2011).

[32] N. J. Poplawski, Phys. Rev. D 83, 084033 (2011).

[33] S. W. Kim, Nuovo Cimento B 112, 363 (1997).

[34] M. Szydlowski and A. Krawiec, Phys. Rev. D 70, 043510 (2004).

[35] J. R. Ray and L. L. Smalley, Phys. Rev. D 27, 1383 (1983).

[36] K. F. Shie, J. M. Nester and H. J. Yo, Phys. Rev. D 78, 023522 (2008).

[37] L. Fabbri and S. Vignolo, Int. J. Theor. Phys. 51, 3186 (2012).

[38] B. Vakili and S. Jalalzadeh, Phys. Lett. B 726, 28 (2013).

[39] K. Atazadeh, JCAP 06, 020 (2014). 